\title{
Short communication \\ Do BIG1-98 and ZOFAST demand a change in guidelines for endocrine therapy?
}

\author{
lan E Smith
}

The Royal Marsden Hospital and Institute of Cancer Research, Fulham Road, London SW3 6JJ, UK

Corresponding author: lan E Smith, ian.smith@rmh.nhs.uk

Published: 18 December 2009

This article is online at

http://breast-cancer-research.com/supplements/11/S3/S18
Breast Cancer Research 2009, 11(Suppl 3):S18

(doi:10.1186/bcr2437)

(c) 2009 BioMed Central Ltd

\section{The BIG1-98 trial}

The BIG1-98 trial, involving over 8,000 postmenopausal women with hormone receptor-positive early breast cancer, was a four-arm trial of adjuvant endocrine therapy for early breast cancer comparing 5 years of tamoxifen treatment versus 5 years of letrozole treatment versus 2 years of tamoxifen then switch to letrozole treatment versus 2 years of letrozole then switch to tamoxifen treatment. The initial results of the trial, which compared only the upfront tamoxifen and letrozole arms, showed a small but highly statistically significant disease-free survival (DFS) advantage in favour of letrozole with a hazard ratio of 0.81 and an absolute 4-year improvement in DFS of $2.7 \%$ [1].

These results were entirely compatible with the other major upfront adjuvant aromatase inhibitor trial, the 'Arimidex', Tamoxifen, Alone or in Combination (ATAC) trial. This was a three-arm trial in which tamoxifen treatment for 5 years was compared with anastrozole treatment for 5 years and compared with the combination of the two treatments. This trial likewise showed a DFS advantage in favour of anastrozole, with a hazard ratio of 0.83 and a 4 -year absolute disease-free survival difference of $2.4 \%$ [2].

Neither the BIG1-98 trial or the ATAC trial showed any significant overall survival benefit at first analysis.

In the BIG1-98 trial update, 4,922 patients were included in the tamoxifen versus letrozole monotherapy comparison [3]. With a median follow-up of 76 months, letrozole treatment continued to show a statistically significant DFS advantage over tamoxifen therapy, with a hazard ratio of 0.88 . In the intent-to treat overall survival analysis there was a very strong trend in favour of letrozole over tamoxifen that did not quite reach statistical significance, with a hazard ratio of 0.7 (95\% confidence interval, 0.75 to 1.02). These results were confounded, however, by $25 \%$ of patients initially randomised to tamoxifen treatment electing to crossover to letrozole treatment in 2005 when the initial results were announced. Most of these patients crossed over in years 3 to 5 with a median duration of letrozole treatment after crossover of 18 months. This led to a bias against letrozole because other data (see below) have shown that a switch from tamoxifen to an aromatase inhibitor after around 2 years is associated with an improved DFS.

In a censored analysis to allow for this bias, the overall survival ratio was significantly in favour of letrozole with a hazard ratio of 0.81 ( $25 \%$ confidence interval, 0.69 to 0.94 ). The true figure for overall survival difference probably lies between these two analyses and would almost certainly show a significant survival benefit for letrozole.

No suggestion of a similar survival advantage has been seen in the ATAC trial, with the most recent hazard ratio for tamoxifen treatment versus anastrozole treatment being 0.97. The Femara versus Anastrozole Clinical Evaluation trial directly compares letrozole with anastrozole in over 4,000 women with node-positive breast cancer, and will provide data on whether letrozole does indeed achieve superior clinical efficacy to anastrozole.

Prior to the BIG1-98 update, several trials (including IES, ARNO, ABCSG and ITA) suggested that sequential adjuvant aromatase inhibitors given after 2 to 3 years of tamoxifen treatment had a significant DFS advantage over tamoxifen alone, with hazard ratios ranging from 0.42 to 0.76 . These hazard ratios appeared superior to those achieved with the upfront aromatase inhibitor approach compared with tamoxifen, and led to speculation that sequential therapy starting with tamoxifen and then switching after around 2 years might be superior to starting with an aromatase inhibitor up front. 
The BIG1-98 trial update also addressed this issue. The trial included for the first time randomised data on the sequential therapy approach. Three blinded arms were compared: letrozole treatment for 5 years versus 2 years of tamoxifen and then switch to letrozole treatment versus 2 years of letrozole and then switch to tamoxifen treatment. This evaluation had a median follow-up of 71 months. Overall there was no significant difference between any of the three treatments in terms of 5-year DFS (87.9\%, 87.6\% and 86.2\%, respectively). Upfront letrozole treatment for 5 years, however, showed a strong trend towards superiority over starting with tamoxifen for 2 years and then switching to 3 years of letrozole treatment. This was particularly seen in patients with nodepositive disease. No such trend was seen in the letrozole for 5 years versus letrozole then tamoxifen comparison.

In conclusion, the BIG1-98 trial update has for the first time shown a very strong trend towards an overall survival advantage in favour of upfront letrozole versus tamoxifen treatment, which would probably have reached significance without the confounding effect of crossover in the tamoxifen arm. The update has also shown no advantage of starting with tamoxifen for 2 years and then switching; indeed, the trend is in the other direction. The evidence from the BIG 1-98 update therefore argues strongly in favour of postmenopausal women with early hormone receptor-positive breast cancer being treated initially with letrozole $2.5 \mathrm{mg}$ orally daily for 5 years in the first instance. It is important to note, however, that tamoxifen still remains a very effective alternative where toxicity or cost issues cause problems with letrozole treatment.

\section{The ZO-FAST trial}

In the ZO-FAST trial 1,060 postmenopausal women with hormone receptor-positive breast cancer being treated with adjuvant letrozole $2.5 \mathrm{mg}$ orally daily were randomised to additional zoledronate $4 \mathrm{mg}$ intravenously 6-monthly for 5 years versus zoledronate added only in the face of the bone mineral density $\mathrm{T}$ score falling below -2 or the development of clinical or asymptomatic fracture at 36 months (so-called delayed treatment). The primary endpoint was the percentage change in lumbar spine bone mineral density at 12 months, but important secondary objectives included the incidence of fractures at 3 years, the time to disease recurrence/relapse, overall survival and safety.

The ZO-FAST trial achieved its primary endpoint demonstrating a lumbar bone mineral density improvement of $4.39 \%$ at 12 months in patients on immediate zoledronate compared with a mean bone mineral density loss of $-4.9 \%$ in those on delayed zoledronate $(P<0.0001)$. Similar changes were seen for the hip. This overall difference in bone mineral density emerged within 12 months of starting therapy and continued to increase during year 2 and again during year 3 .

Intriguingly, patients on immediate zoledronate treatment also had a significantly decreased risk of DFS compared with those on elective delayed therapy, with a hazard ratio of 0.59 $(P=0.03)$. This included a lower incidence of local recurrence $(0.4 \%$ for immediate zoledronate versus $1.9 \%$ recurrence for delayed zoledronate), suggesting the effect was not simply mediated through inhibition of bone metastases (where the difference was $1.7 \%$ vs. $3.2 \%$, respectively). So far $2.1 \%$ of patients have died following immediate zoledronate treatment, versus $3.4 \%$ in the delayed arm. There was no significant increased risk of adverse events with the immediate treatment approach. No cases of renal impairment related to the study drug were seen. Osteonecrosis of the jaw was seen in one patient $(0.2 \%)$ on immediate zoledronate treatment versus none with the delayed approach.

Further evidence for an overall improvement in DFS with immediate 6-monthly zoledronate comes from the ABCCSG-12 trial [4]. In this trial 1,803 premenopausal women were randomised to tamoxifen and goserelin treatment versus anastrozole and goserelin treatment. In a secondary randomisation, all patients were also randomised to zoledronate treatment versus no additional bisphosphonate therapy.

With 48 months median follow-up, the addition of 6-month zoledronate therapy prevented bone mineral density loss, which was seen in both endocrine therapy arms without zoledronate.

In addition there was again an improvement in DFS for patients randomised to receive immediate 6-monthly zoledronate with a hazard ratio of $0.64(P=0.019)$ compared with the delayed approach. As in the ZO-FAST trial, there was a reduction in local regional relapse (10 patients vs. 20 patients) as well as in distant relapse including bone metastases (23 patients vs. 16 patients). Sixteen patients have so far died in those treated with zoledronate, versus 26 patients in those without this treatment.

Zoledronate has several potential mechanisms of action to explain this observation, including the induction of apoptosis, the inhibition of tumour cell growth in vitro, inhibition of adhesion and invasion, and possible anti-angiogenic effects.

Current National Institute of Clinical Excellence guidelines and St Gallen recommendations suggest that bisphosphonates should not be used routinely as adjuvant therapy, but should be guided by bone mineral density.

Although the ZO-FAST trial results are from early phases, the fact that they are reinforced by very similar results from the ABCCG-12 trial argues that current guidelines should be changed and that zoledronate treatment by 6-monthly intravenous infusion should be included as standard therapy for women with hormone receptor-positive early breast cancer. This inclusion of intravenous zoledronate is not just to prevent bone mineral density loss but also on the basis of a significant DFS advantage. 


\section{Competing interests}

The author declares that they have no competing interests.

\section{Acknowledgement}

This article has been published as part of Breast Cancer Research Volume 11 Suppl 3 2009: Controversies in Breast Cancer 2009. The full contents of the supplement are available online at http://breastcancer-research.com/supplements/11/S3.

\section{References}

1. TBreast International Group (BIG) 1-98 Collaborative Group, Thürlimann B, Keshaviah A, Coates AS, Mouridsen H, Mauriac L, Forbes JF, Paridaens R, Castiglione-Gertsch M, Gelber RD, Rabaglio M, Smith I, Wardley A, Price KN, Goldhirsch A: A comparison of letrozole and tamoxifen in postmenopausal women with early breast cancer. N Engl J Med 2005, 353:2747-2757.

2. Duffy $S$, Jackson TL, Lansdown M, Philips K, Wells M, Pollard S, Clack G, Coibion M, Bianco AR: The ATAC ('Arimidex', Tamoxifen, Alone or in Combination) adjuvant breast cancer trial: first results of the endometrial sub-protocol following 2 years of treatment. Hum Reprod 2006, 21:545-553.

3. BIG 1-98 Collaborative Group, Mouridsen H, Giobbie-Hurder A, Goldhirsch A,Thürlimann B, Paridaens R, Smith I, Mauriac L, Forbes JF, Price KN, Regan MM,Gelber RD, Coates AS: Letrozole therapy alone or in sequence with tamoxifen in women with breast cancer. N Engl J Med 2009, 361:766-776.

4. BIG 1-98 Collaborative Group, Mouridsen H, Giobbie-Hurder A, Goldhirsch A,Thürlimann B, Paridaens R, Smith I, Mauriac L, Forbes JF, Price KN, Regan MM,Gelber RD, Coates AS: Endocrine therapy plus zoledronic acid in premenopausal breast cancer. N Engl J Med 2009, 360:679-691. 